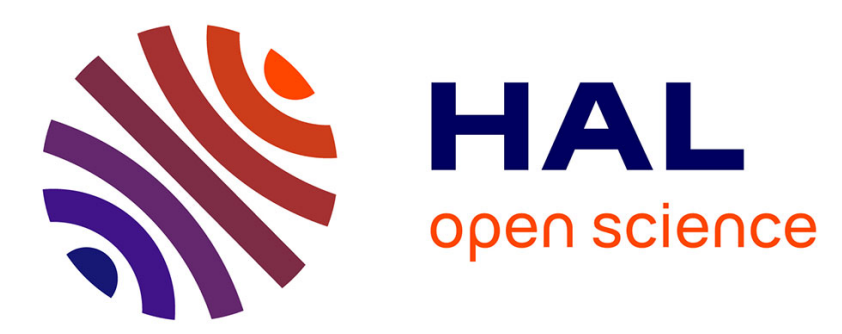

\title{
Elastic characterization of polymer fibers by laser Doppler vibrometry
}

\author{
Ludovic Labelle, N.B. Roozen, Jan Vandenbroeck, Shuichi Akasaka, Christ
}

Glorieux

\section{- To cite this version:}

Ludovic Labelle, N.B. Roozen, Jan Vandenbroeck, Shuichi Akasaka, Christ Glorieux. Elastic characterization of polymer fibers by laser Doppler vibrometry. Optics and Lasers in Engineering, 2017, 99, pp.88-97. 10.1016/j.optlaseng.2016.11.020 . hal-02895869

\section{HAL Id: hal-02895869 https://hal.science/hal-02895869}

Submitted on 10 Jul 2020

HAL is a multi-disciplinary open access archive for the deposit and dissemination of scientific research documents, whether they are published or not. The documents may come from teaching and research institutions in France or abroad, or from public or private research centers.
L'archive ouverte pluridisciplinaire HAL, est destinée au dépôt et à la diffusion de documents scientifiques de niveau recherche, publiés ou non, émanant des établissements d'enseignement et de recherche français ou étrangers, des laboratoires publics ou privés. 


\title{
Elastic characterization of polymer fibers by laser Doppler vibrometry
}

\author{
Ludovic Labellea, N. B. Roozena, Jan Vandenbroeck ${ }^{b}$, Shuichi Akasakac, Christ Glorieux ${ }^{a}$ \\ ${ }^{a}$ Laboratory of Acoustics, Division of Soft Matter and Biophysics, Department of Physics and \\ Astronomy, KU Leuven, Celestijnenlaan 200D, B3001 Heverlee, Belgium \\ ${ }^{b}$ Computational Physics \& Foam Technology, Core Science Group, Huntsman Polyamides, Everslaan \\ 45, B3078 Everberg, Belgium
}

'Tokyo Institute of Technology School of Materials and Chemical Technology, Tokyo, Japan

\section{Introduction}

Automotive, furniture, seating and construction elements include many parts with a functionality serving safety and comfort. In view of requirements concerning shock absorption, vibration control, noise control, sound insulation, and thermal insulation, there is a great need for understanding and accordingly tuning the viscoelastic behavior of the used materials. Globally, visco-elastic characterization of porous material has shown a renewed interest since a few years [1][2][3][4]. In the process of product development and improvement, characterization of the viscoelastic properties of these materials plays a crucial role.

Many materials that are used in applications are porous. The turn-over of the industry producing these materials is significant, and the technical requirements that are requested are becoming stricter. For these reasons there is a significant demand from industry to develop fundamental physical models to predict the behavior of foam materials. Due to the complex formation process and resulting 3D network structure, there is a particular need to unravel and understand the relation between their microscopic structure and macroscopic elastic behavior. By feeding back this information to the development and manufacturing process, the product properties can be optimized for specific applications. The complexity of porous structures makes it an interesting challenge to predict their technical properties and physical behavior at macroscopic scale, based on their 3D morphology and microscopic behavior. This can be achieved by numerical (finite element) modeling, in which the found microscopic elastic properties are used for the microscopic elements of discretized morphological foam structures that are obtained by microCT. The vibrational response of the macroscopic structure is then obtained by mechanically exciting it at the subsonic and audio frequencies of interest, for which the wavelength is much larger than the pore size. From the dynamically changing macroscopic strain map, the compressibility of the effective medium, or the wavelength of the travelling waves can be determined.

The first step in mapping the elastic behavior of a porous material on a microscopic scale is to determine the elastic moduli, i.e. the Young's modulus and Poisson's ratio (or equivalent quantities such as the longitudinal and transverse wave velocity and the Lamé coefficients), of single struts of the frame. The next step is the characterization of strut connections, or nodes, and the membranes. The 
incentive behind the separate elastic characterization of these different categories is that their elasticity is expected to be different. The fact that struts, nodes and membranes have different geometries and dimensions suggests that they have been undergoing a different history of formation. In particular, due to the thermal and chemical processes involved they have vitrified at different moments during the formation process. As a result, the chemical reactions involved in their polymerization have stalled at different phases in the chemical conversion process, making their state of polymerization, and thus their elastic relaxation behavior different from the one in bulk, and different amongst each other. Since struts, nodes and membranes all contribute to the global macroscopic elasticity, it is of great importance to get a view on the differences in their elastic behavior.

This paper fits in a research project whose ultimate goal is to determine the frequency dependence of elastic moduli of microscopic foam structures from reliably measured dispersion curves. The followed approach is to use Laser Doppler Vibrometry (LDV) to detect piezoelectrically and laser excited elastic wave displacement patterns in microscopic foam structures. This aim involves several interesting challenges to be tackled: (i) efficient excitation and sensitive detection of waves along thin fibers with high spatial and temporal resolution, (ii) unraveling the frequency dependence of the relaxing material and the one due to the finite dimensions of the structure in which the guided waves are travelling, (iii) coping with the complexity of multiple reflections of guided waves travelling in the network of interconnected fibers.

The intention of this paper is to characterize polymer fibers by means of laser Doppler vibrometry. The approach presented in this paper is applied to a nylon fiber with a diameter of approximately $100 \mu \mathrm{m}$ and a rectangular polyamide fiber grid with dimensions in the $0.1 \mathrm{~mm}-100 \mathrm{~mm}$ range. These samples were chosen on the basis of the similarity of their geometry with the one of struts and networks in foams, while having dimensions that are larger, making the experimental proof of concept of the proposed approach more simple. The dispersion curves are analyzed with respect to the influence of the shape of the fiber cross section and of the complex structure in which the fiber of interest is embedded. They are fitted with analytical and numerical models to obtain the Young's modulus of the samples. The resulting values for the Young's modulus are compared with data from Dynamical Mechanical Thermal Analysis (DMTA) and from literature.

This paper presents new experimentally obtained dispersion relationships of fiber wires and fiber grids, which are compared with numerically obtained results. These results are relevant in the context of the overall objective of this work to make a step towards microscale characterization of foam structures. These samples were chosen on the basis of the similarity of their geometry with the one of struts and networks in foams, while having dimensions that are larger, making the experimental proof of concept of the proposed approach more simple. The dispersion curves are analyzed with respect to the influence of the shape of the fiber cross section and of the complex structure in which the fiber of interest is embedded. Although much simpler, similar to foams, the cubic grid is characterized by crossconnections, which result in discontinuities along the scanning path when the path is chosen long enough to allow to analyse long wavelengths and low frequencies. The next step towards realistic foams, using a 3D printed polymer grid with randomly oriented and connected struts, will be the subject of future investigations.

\section{Experimental details}


Two samples were experimentally investigated. Sample 1[Figure H1]was a nylon wire with a circular cross section and a diameter of about $150 \mu \mathrm{m}$ and a length of $50 \mathrm{~mm}$. The fiber was rigidly clamped at one end. Tension in the wire was provided by a little ring with a weight of $18 \mathrm{~g}$, pending on the other end.

Sample 2 [Figure H2] was a 3D printed, rigid polyamide (PA) grid structure with a regular square-like cross section and strut thickness of $1.1 \mathrm{~mm}$.

Both for the nylon fiber and for the PA grid, we have made use of photoacoustic excitation (Figure 1a) of guided elastic waves by illuminating a spot on the sample with a laser beam from a pulsed laser. Although in the visible range the nylon fiber was transparent and the PA grid was white, the optical absorption for the laser wavelength $(1064 \mathrm{~nm}$ from a $10 \mathrm{~ns}$ Spectra Physics Quantaray Nd:YAG laser working at $10 \mathrm{~Hz}$ repetition rate or $1057 \mathrm{~nm}$ for a 10ps High Q Nd:YLF laser working at $1 \mathrm{kHz}$ repetition rate) was sufficient to generate sufficient heating and thermal expansion and launch guided elastic waves that were measurable in the $50 \mathrm{kHz}-2.5 \mathrm{MHz}$ range.

For the nylon fiber sample we have also made use of piezoelectric excitation (Figure 1b).The electromechanical conversion efficiency of piezoelectric transducers is mainly high when they are used in resonance. In order to cover the frequency range from $\mathrm{Hz}-\mathrm{MHz}$, we have made used of a clamped piezo-metal bilayer-membrane configuration, with a large bending efficiency, with a substantial number of membrane resonances in the $1 \mathrm{kHz}-100 \mathrm{kHz}$ range.

The transducer consisted of a PZT-disk of $10 \mathrm{~mm}$ diameter and $0.5 \mathrm{~mm}$ thickness, glued on a circular steel membrane of $15 \mathrm{~mm}$ diameter and $1 \mathrm{~mm}$ thickness, which was clamped at its edges in a PVC ring.

By applying a sinusoidal voltage with 50Volt amplitude, generated by a computer controlled homemade sine wave generator and amplified by a power generator ENI AP400B, bending modes of the bilayer membrane could be efficiently excited, with maximum displacement amplitudes at resonances in the $50 \mathrm{kHz}-700 \mathrm{kHz}$ range ranging between $60 \mathrm{~nm}$ and $1600 \mathrm{~nm}$ (Figure 2). The reason to limit the frequency range to the range mentioned is that the structural resonances of the piezoelement and its membrane lie in this range. The transducer vibrations were transferred to the sample by a needle $(0.5 \mathrm{~mm}$ diameter, $4 \mathrm{~mm}$ length), which was standing perpendicular to the transducer surface, whose bottom was to the transducer, and whose tip was touching the nylon fiber.
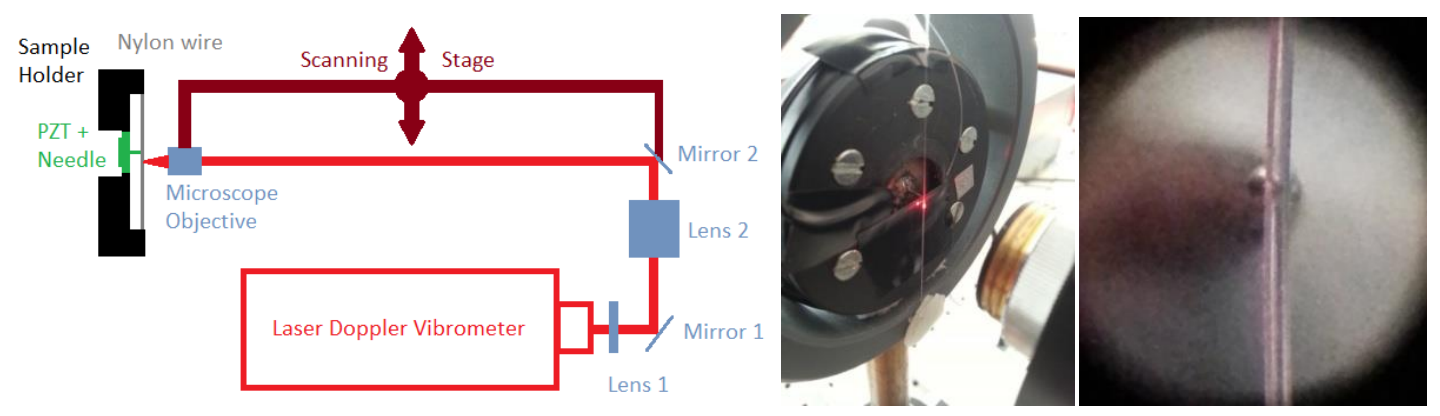

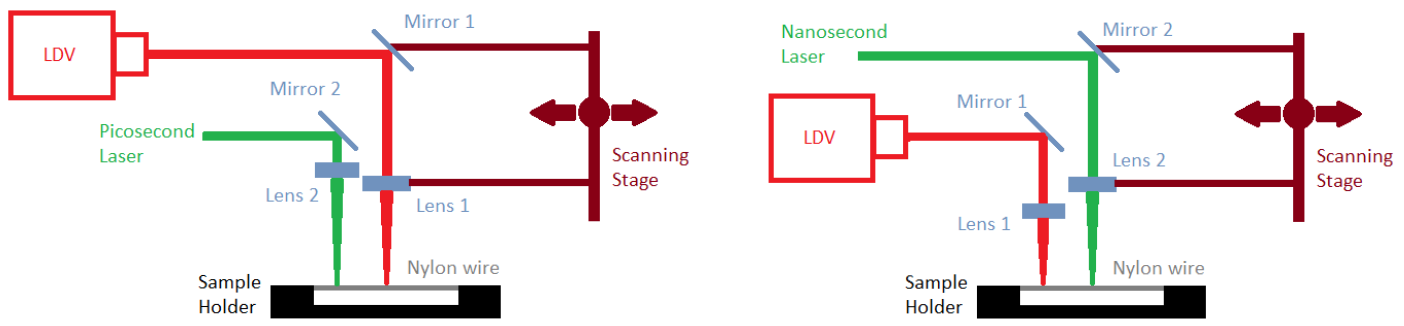

Figure 1: Experimental setups: Top left: Nylon wire measurement setup making use of PZT + needle excitation at a fixed point and scanning with the probe LDV. Top middle: Photo of the actual nylon wire mounted on a PVC support, excited by PZT + needle, also showing the LDV probe spot focused on the sample with microscope objective. Top right: Photo of the view through the microscope objective showing the nylon wire in contact with the needle tip. Bottom left: Nylon wire setup with picosecond laser excitation at a fixed position and scanning with the probe LDV. Bottom right: Nylon wire setup with scanning nanosecond laser excitation and the probe LDV at a fixed position.

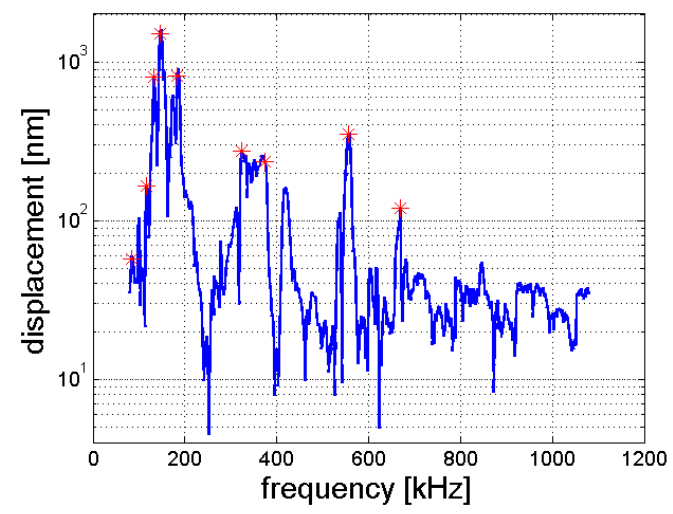

Figure 2: Spectrum of the axial displacement of the needle tip during excitation of the piezoelectric actuator excited with $50 V_{\mathrm{pp}}$ amplitude.

Both in the case of photoacoustic and piezoelectric excitation, a Laser Doppler Vibrometer (Polytec controller and sensor head OFV-353) was used to detect the displacement field (wave pattern) along the sample. A scanning stage from APT Thorlabs was used to scan either the LDV beam or the exciting $\mathrm{Nd}$ :YAG laser beam along the sample. Signals were recorded on an oscilloscope (Lecroy LC564A) and acquired by a Labview program for every positions of the scan.

The LDV displacement detection sensitivity was $50 \mathrm{~nm} / \mathrm{V}$ for vibration frequencies above $20 \mathrm{kHz}$. The displacements generated by piezoelectric transducers vary from millimeters in the $\mathrm{Hz}$ range till nanometers in the $\mathrm{MHz}$ range. Laser Doppler vibrometry combines the unique capacity to cover this enormous dynamic range with excellent linearity with the possibility to remotely scan the probed location with micrometric spatial resolution along arbitrary paths.

In the case of needle excitation, the discrete excitation frequencies were chosen to be the resonance frequency of the assembly (transducer + metal membrane): 84, 117, 132, 146, 184, 323, 374, 556, $669 \mathrm{kHz}$. For each frequency, a position scan was performed. A Fourier transform was used to compute the response of the wire in the frequency domain. In order to optimize the measurement settings 
(scope amplitude range and time range), the measurement was split in two parts: (i) Scanning range of $12 \mathrm{~mm}$ and the scanning step $0.1 \mathrm{~mm}$ for the lower five frequencies tested (scan 1), (ii)Scanning range of $10 \mathrm{~mm}$ and the scanning step of $0.05 \mathrm{~mm}$ for the four higher frequencies tested (scan 2).

The phase velocity dispersion curve, from which the elastic modulus of the sample material was fitted, was extracted from the axial position dependence of the displacement signals. Two position scanning (in steps of 100microns) strategies were followed. (i) Scanning the point of incidence of the pump laser spot (with a spherical lens laser dot) along the fiber or rod, while keeping the probe laser spot (150micron diameter) at the same location on the sample. This configuration allowed making use of a small piece of retroreflective tape on the detection spot for enhancing the amount of backscattered probe light and thus the signal to noise ratio. (ii) Keeping the Nd:YAG pump laser spot at the same location, while scanning the probe laser spot axially along the sample. The advantage of keeping the laser pump position fixed is that material damage due to repeated illumination by high energy pulses remained local, without effect on most of the path trespassed by the guided acoustic waves.

For piezoelectric excitation by the needle contacting the nylon fiber, probe laser scanning was the only option, since the quality of the mechanical contact between the needle and the sample was quite delicate. For this configuration, in view of the optically poorly reflecting surface of the nylon fiber sample, the scan was extended to the angular direction across the fiber, in order to increase the number of detection locations, thus enhancing the probability of finding locations with good backscatter of the probe beam into the vibrometer, thus obtaining signals with good S/N. For every axial position, an average of the five data along the scan across was determined, thus obtaining one value per axial position for further analysis.

\section{Results and discussion}

As mentioned above, this investigation was performed in a wider context of developing excitation and detection techniques to determine a dispersion curve and therefrom the viscoelastic properties of polymer fibers in polyamide foams. The phase velocity dispersion curve is rich in information but the information is difficult to extract, because of the joint influence of geometry induced (guided wave dispersive behavior) and material relaxation (elastic moduli) induced frequency dependence. Access to a wide bandwidth is important to assess as much as possible of the frequency range over which relaxation is changing the viscoelastic properties, stiffening the material at high frequencies (and low temperatures) and softening it at low frequencies (and high temperatures). An important challenge is therefore to excite and detect with high spatial resolution over sufficiently long distances, so that both the short wavelength (high frequency) and long wavelength (low frequency) part of the dispersion curve can be determined.

\subsection{Nylon fiber}

As a first step tackling this challenge, a nylon wire was used as a simple model system, with well-known elastic properties, and dimensions that are intermediate between the ones of systems that are used 
in classical ultrasonic non-destructive testing, and the ones of the struts in the complex porous materials that we are interested in. In the frequency range that could be accessed by our experimental methods, and at room temperature, the frequency dependence of the elastic properties of nylon is moderate, with the real part of Young's modulus evolving from 5.7GPa in the low part of the audio frequency range, over $6 \mathrm{GPa}$ in the $\mathrm{kHz}$ range, to 6.4GPa at $\mathrm{MHz}$ frequencies (Figure 3). The curves in Figure 3 are based on DMTA measurements carried out on a bundle of 10 fibers, which was fixed between two clamps spaced by $10 \mathrm{~mm}$, in a frequency range between $1-50 \mathrm{~Hz}$ at different temperatures, and exploit the time-temperature superposition principle that is known for relaxing materials [5][6][7]. The curves were extended by shifting (by applying suitably chosen horizontal shift factors) the data until a so-called "master"-curve for a desired temperature (e.g. room temperature of $25^{\circ} \mathrm{C}$ ) is established. One possible approach for determining the shift factor is to make use of the Williams-Landel-Ferry model [8].The imaginary part of Young's modulus is about $2.5 \%$ of the real part of Young's modulus in the frequency range of interest (from a few $\mathrm{kHz}$ up to $\mathrm{MHz}$ frequencies, illustrated by red lines).
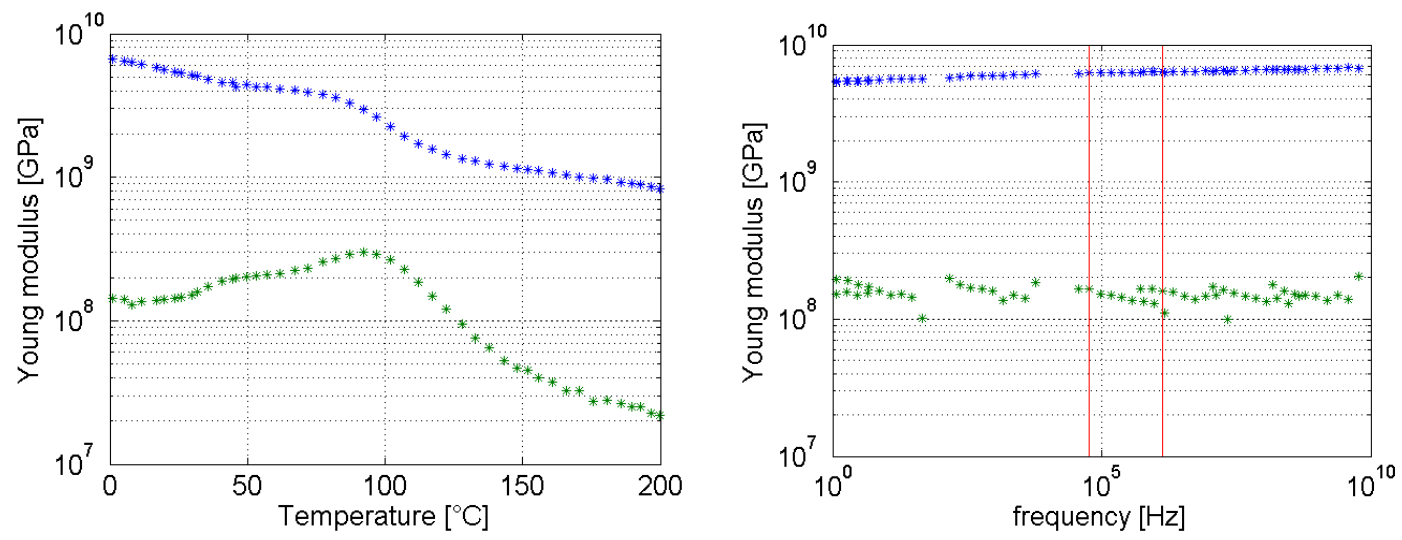

Figure 3: (left): Temperature dependence of the real (blue curve) and imaginary (green curve) part of the Young's modulus of a nylon wire, measured by DMTA on a bundle of 10 fibers, exciting the sample at a fixed

frequency of $25 \mathrm{~Hz}$. (right) : converted DMTA results showing the frequency dependence of the Young's modulus (blue curve: real part, green curve: imaginary part) of a nylon wire, at a fixed temperature of $25^{\circ} \mathrm{C}$. Frequency range of interest in the measurements is indicated between red vertical lines.

The $50 \mathrm{~mm}$ length of the nylon fiber was suitable to perform position scans with sufficient length and thus also a sufficient wavenumber domain resolution. This measurement allowed verifying the quality of the dispersion curves both for wavelengths shorter and longer than the typical strut length $(\cong 100 \mu \mathrm{m})$ in porous materials, without complications of complex wave interactions at strut interconnections in the porous network. Analyzing wavelengths in the $\mathrm{mm}$ range, hence longer than typical strut lengths, is important for investigating foams in at vibration and audio frequencies.

\subsubsection{Piezo-needle excitation}

Figure 4 shows the frequency domain results (real and imaginary parts) of the wave induced displacement pattern along the axial direction at different discrete, piezo-needle excitation frequencies. The displacement field quality and amplitude vary along the direction across the fiber, making it worth to perform the cross scan, and average or select the best data. The patterns were measured by scanning the probe laser spot along the wire in steps of 100 microns. Steps were also 
made in the direction perpendicular to the axial direction to search for optimal measurement position in terms the reflection of the laser beam, and thus the signal-to-noise ratio of the LDV-signal.
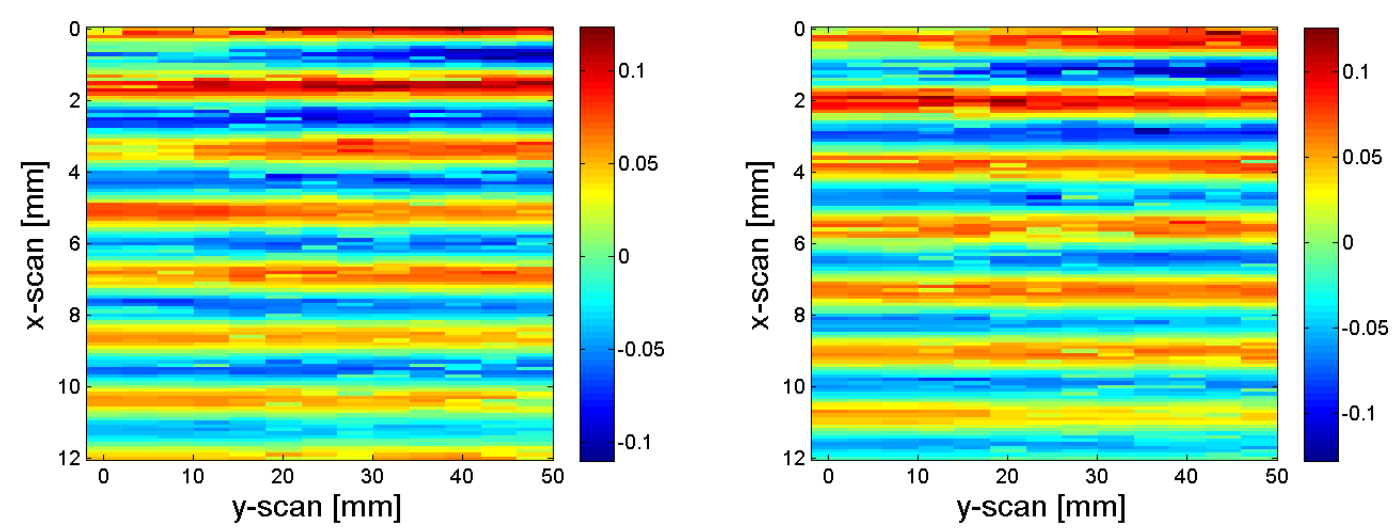

Figure 4: Snapshot of the real (left) and imaginary (right) part 2D LDV position scan measurement result (100 micron steps) for sinusoidal piezo-needle excitation on a fixed location nylon wire at $184 \mathrm{kHz}$. The wave propagation direction is along the vertical axis. The color bar indicates the displacement scale in $\mathrm{nm} / \mathrm{a} . \mathrm{u}$.

For the sake of seeking for computational simplification when fitting dispersion curves of fibers with sometimes complex cross sectional shapes, we have verified by finite element calculations to what extent the shape of the cross section of the nylon wire is affecting the dispersion curve, compared to an infinite plate. With this knowledge, given a dispersion curve for a certain wire cross section shape, the value of Young's modulus can be first fitted in a fast way by using a semi-analytical model for a plate. This has the important advantage in that, compared to an approach in which the direct calculation is performed numerically, a semi-analytical model for a plate can be very easily implemented (programmed) in a multi-parameter fitting problem, with fast calculations. Next, the known adaptation factor for the shape can be used to obtain the true value.

Table T1 lists values of the equivalent Young's modulus of an infinite plate, obtained by fitting the numerically calculated dispersion curves on to the plate model. A bulk Young's modulus of of 4GPa and a Poisson's ratio of 0.4 were using in the numerical simulations. The fits were done for wires with a square, circular and triangular cross section (see Fig. 5), while using a semi-analytical infinite plate model. Due to experiencing less motion constriction on their sides, wires are more flexible than semiinfinite plates. As a consequence, fitting the wire dispersion curve with a plate model results in a lower value of Young's modulus. The triangular shape being less massive than the circular one, and even more than the square one, explains the order of the deviations: $28 \%$ Young's modulus reduction for the triangular shape, while only $15 \%$ for the circular shape and as little as $5 \%$ for the square shape. Note that latter deviation is different from the Poisson effect as predicted by the low-frequency EulerBernoulli theory, which is known to be equal to $\left(1-v^{2}\right)=0.84(=-16 \%)$ [9], with $v=0.4$ the Poisson ratio of the fiber material that was used to calculate the dispersion curves.

In the remainder of this paper, we will use the found deviations as adaptation factors to convert values of Young's modulus, determined by fitting (with limited computational effort) with an analytical plate model, to estimates of the value that would be obtained (with very large computational load) using the numerical model that takes into account the shape details. 
As mentioned earlier, from the DMTA analysis of the nylon fiber, the dispersive Young's modulus was found to be weakly dispersive, with a variation between $5.7 \mathrm{GPa}$ and $6.4 \mathrm{GPa}$ in the $5 \mathrm{kHz}-1 \mathrm{MHz}$ range. In view of that, although the analysis allows to extract a frequency dependent Young's modulus values from the phase velocity data, we have fitted the dispersion curves assuming a frequency independent Young's modulus. Fitting both the Young's modulus and Poisson's ratio would require access to the high frequency Rayleigh wave limit of the dispersion curve. Since for nylon the guided waves above $2 \mathrm{MHz}$ were too damped to extract a phase velocity, the available part of the experimentally determined dispersion curve is still in the low frequency limit of flexural wave behavior. Therefore, for fitting with an analytical plate model, we have assumed ad hoc a Poisson's ratio of 0.4 .

In order to get more insight in the effect of the wire cross section geometry on the guided wave propagation, we have also compared the dispersion curves calculated for the wires with different shapes but having the same cross section area (Figure 5), and thus the same mass per unit of length. The relative difference in the value of Young's modulus for which the dispersion curve calculated by numerical fiber models are matching the dispersion curve calculated by the analytical model of an infinite plate with thickness equal to the characteristic dimension D (full black lines, Fig 5), are presented. In order to make Young's modulus fitted from the dispersion curve of a circular wire using a square cross section model matching the correct value, the circle diameter has to be increased with $13 \%$ compared to the one of the circle with cross section equal to the one of the square wire. The height of the triangle has to be increased with $30 \%$. The large corrections infer that the linear density of the wire is not an adequate measure to compare the flexural stiffness of different cross section shapes.

For a comprehensive treatment of the effect of geometry on the dispersion of flexural waves, we refer to [10].

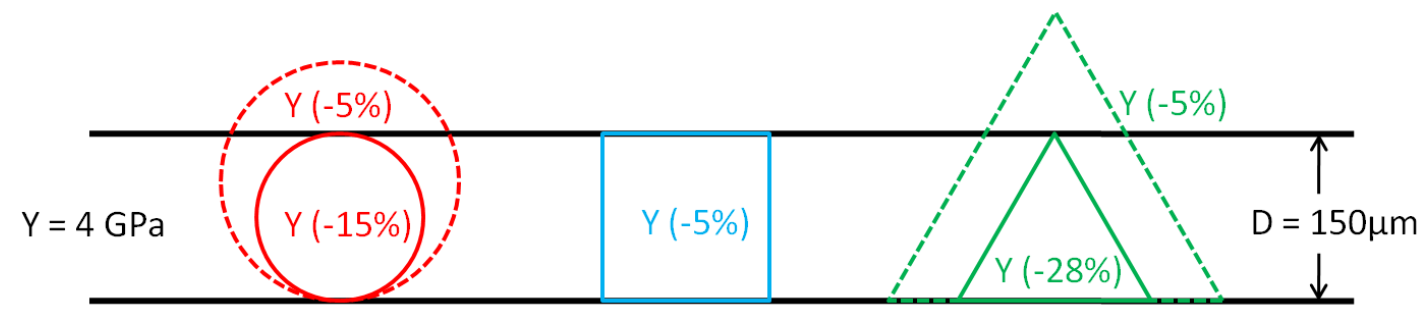

Figure 5: Scheme of the analytical infinite plate model of 150 micron thickness (full black lines) and numerical fiber models with circular (full red line), square (full blue lines) and triangular (full green lines) cross section surface. Also enlarged circle and triangle cross sections are shown, with cross section surface equal to the one of the square cross section.

Table T1. Fit results on to a plate model, for various cross section shapes.

\begin{tabular}{|c|c|c|c|}
\hline cross section shape & \multicolumn{3}{|c|}{ Young's modulus (GPa) } \\
\cline { 2 - 4 } & $\begin{array}{c}\text { Fitting result with } \\
\text { infinite plate }\end{array}$ & Deviation & $\begin{array}{c}\text { Thickness } \\
\text { correction }\end{array}$ \\
\hline square & $3.8 \pm 0.2$ & $-5 \%$ & $0 \%$ \\
\hline triangular & $2.9 \pm 0.4$ & $-28 \%$ & $30 \%$ \\
\hline circular & $3.4 \pm 0.2$ & $-15 \%$ & $13 \%$ \\
\hline
\end{tabular}


We have also verified by an analytical simulation to which extent the tension of in a nylon wire of $\phi_{\text {wire }}=150$ micron diameter, caused by pulling it by a suspended mass $\mathrm{m}=7 \mathrm{gram}$ in order to keep it stretched and stable, affects the dispersion curve. Figure 6 shows the effect of the tensile stress $\sigma=\mathrm{T} / \mathrm{A}=\mathrm{mg} / \mathrm{A}=0.14 \mathrm{~N} /\left(\pi \phi_{\text {wire }}{ }^{2} / 4\right)=0.025 \mathrm{GPa}$, with $\mathrm{T}$ the tensile force and $\mathrm{g}=9.81 \mathrm{~m} . \mathrm{s}^{-2}$ the gravitational acceleration, on the phase velocity in the nylon wire. The results show that the tensile stress $\sigma=T / A$, which is much smaller than $Y I k^{2} / A$ (see Eq. 1a), starts to have a noticeable effect on the dispersion curve for frequencies below $18 \mathrm{kHz}$ (using $Y=6 \mathrm{Gpa}, R=75 \mu \mathrm{m}$, and $k$ as follows from Eq. 1a). At high frequencies the stiffness of a wave is dominated by the bending effect of the wire. And the tension effect is negligible as compared to the bending stiffness. This makes that the curves coincide at higher frequencies.

At $10 \mathrm{kHz}$, the effect of tension is still significant. For a nylon wire with geometric dimensions, material parameters and tensile force considered, the effect is negligible at frequencies of $100 \mathrm{kHz}$ and higher [11].

The phase velocity $c_{\text {tension }}$ was calculated with the following parameters and formula (taken from [15]): radius $R=75 \mu \mathrm{m}$, tensile force $T$, density $\rho=1150 \mathrm{~kg} / \mathrm{m}^{3}$, linear density $\rho_{L}=\rho \pi R^{2}$, angular frequency $\omega=2 \pi f$, frequency $\mathrm{f}$, wave number $k=\omega / c$, Young's modulus $Y=6 \mathrm{GPa}$, second moment of area of a circle cross section $I=\frac{\pi R^{4}}{4}$.

$$
\omega=k * c_{\text {tension }} \sqrt{1+\frac{Y * I * k^{2}}{T}}
$$

In the case of a stress free wire, the equation reduces to a simple power law, with the phase velocity only depending on the Young's modulus, the linear density and the radius (second part of equation valid for a wire with a circular cross-section)

$$
c(\omega)=\left(\frac{Y I}{\rho_{L}} \omega^{2}\right)^{\frac{1}{4}}=\left(\frac{Y}{\rho_{L}}\left(\frac{R \omega}{2}\right)^{2}\right)^{\frac{1}{4}}
$$

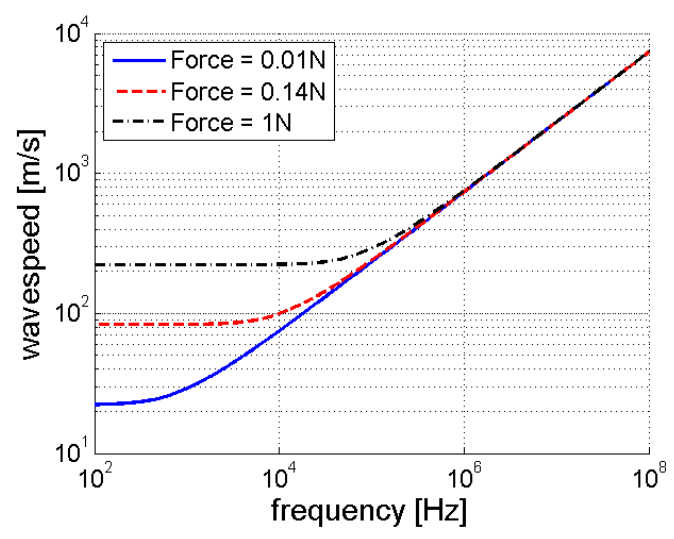

Figure 6: Effect of tensile force on phase velocity on a nylon wire as predicted by equation (Eq.1) 

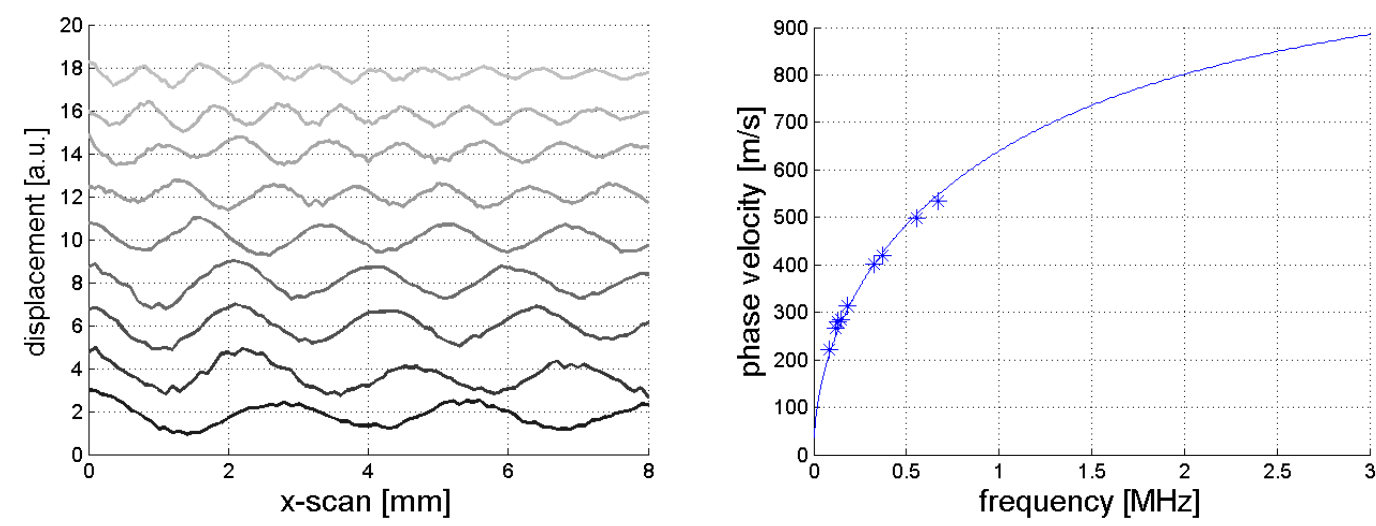

Figure 7: Left: Axial dependence of the guided wave displacement along the nylon fiber, for sinusoidal piezoelectric excitation at $84,117,132,146,184,323,374,556,669 \mathrm{kHz}$. Each curve is shifted with an arbitrary value to easily distinguish between them. Right: Experimental data (symbols) and fit with analytical infinite plate model (full line) of $150 \mu \mathrm{m}$ thickness and $Y_{\text {equivalent plate }}=(3.9 \pm 0.1) \mathrm{GPa}$, leading, after shape adaptation correction to $Y_{\text {wire }}=(4.5 \pm 0.1) \mathrm{GPa}$.

Figure 7 (left) shows the real part of the guided wave displacement, exciting the nylon fiber with a single frequency sinusoidal excitation by means of a piezo element with needle. From these patterns, the wavelength was determined by searching for the peak in the wavenumber spectrum using a spatial Fourier transform. Figure 7 (right) shows the resulting phase velocity values. The dispersion curve is well fitted by a semi-analytical plate model with a resulting value for Young's modulus of $Y_{\text {equivalent plate }}=$ $(3.9 \pm 0.1) \mathrm{GPa}$, and, after shape adaptation correction (15\% for plate to circle) to $Y_{\text {wire }}=(4.5 \pm 0.1) \mathrm{GPa}$.

\subsubsection{Photoacoustic excitation}

An interesting possibility to excite elastic waves in a material is offered by the photoacoustic effect, which occurs when it is dynamically illuminated by intensive light. The fraction of the light that is optically absorbed is converted into heat. The resulting increase of temperature leads to thermal expansion and the generation of stress. In case of modulated illumination in general and impulsive excitation in particular, the change of stress is propagating in the form of elastic/acoustic waves in the material and its surroundings.

The contactless nature of photoacoustic excitation of elastic waves, together with the remote probing feature of laser Doppler vibrometry, and the very large bandwidth (in the temporal domain determined by the laser pulse duration and in the spatial domain determined by the laser spot size) makes the approach nicely complementary with piezo-excitation. The excitation geometry can be arbitrarily tuned by choosing the illumination pattern, allowing easy scanning possibilities. In the case of experimental configurations that require scanning of the pump-probe distance, this offers the possibility to keep the detection location, and therefore the probing sensitivity, fixed, while the pumpprobe distance is scanned by moving the pump beam. 

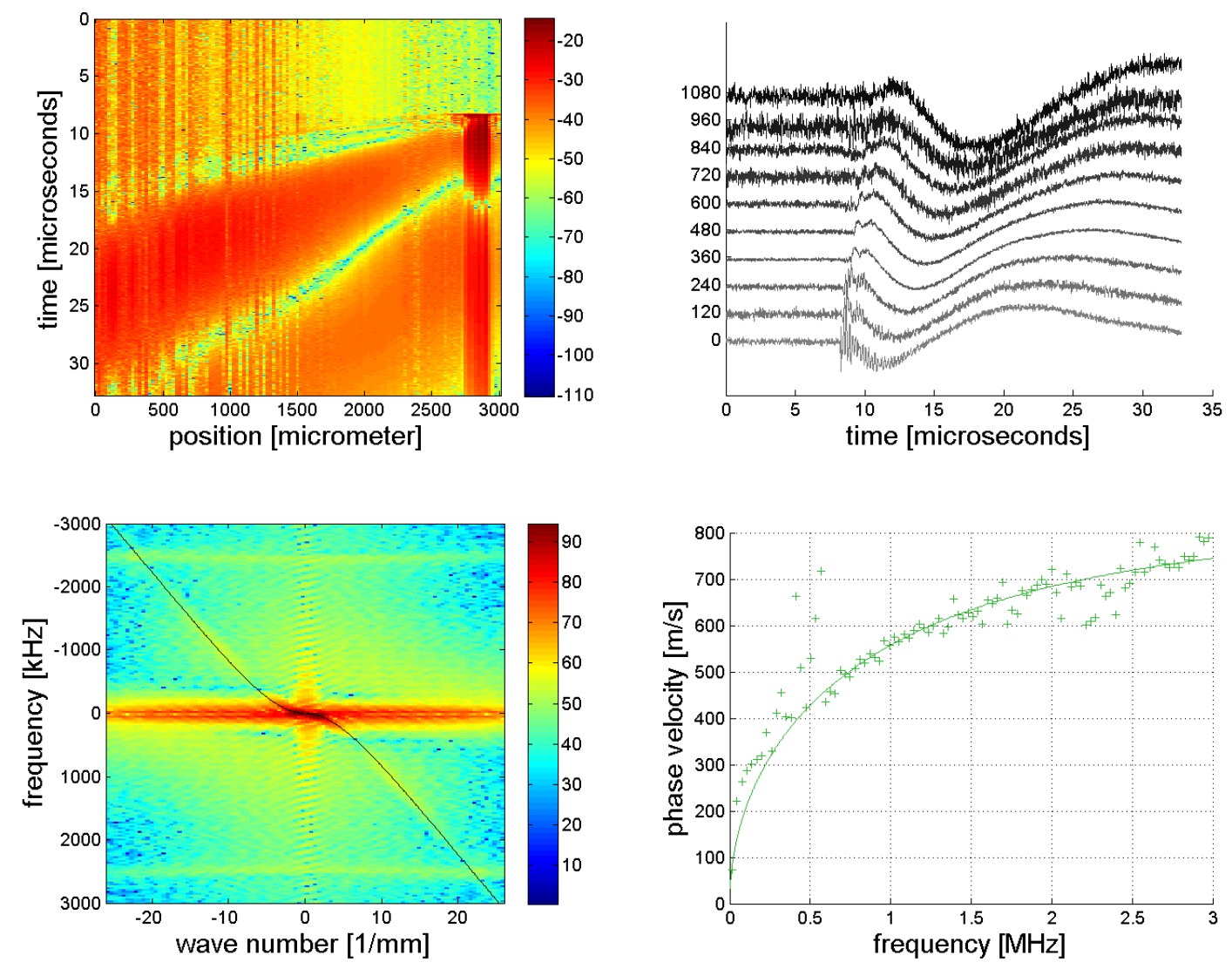

Figure 8: Displacement signals, photoacoustically generated by focused laser pulses of 10ps duration on a 150 micron diameter nylon fiber and detected by a laser Doppler vibrometer, versus pump-probe distance. Top Left: 2D representation of the displacement in a.u. versus time and pump-probe distance. Top Right: time signals recorded at positions spaced by 120 micron. Each curve is shifted with an arbitrary value to easily distinguish between them. Bottom left: spectral amplitude in the frequency-wavenumber domain of the photoacoustically induced displacement field in a 150 micron nylon wire. The full line represents a simulation using a plate model. Bottom right: corresponding phase velocity dispersion curve, and fit with analytical infinite plate model (full line) of $150 \mu \mathrm{m}$ thickness and $Y_{\text {equivalent plate }}=(2.54 \pm 0.07) \mathrm{GPa}$, resulting, after geometrical adaptation, to $Y_{\text {wire }}=(2.92 \pm 0.07) \mathrm{GPa}$.

Figure 8 depicts displacement signals, photoacoustically generated by focused laser pulses of $10 \mathrm{ps}$ duration on a 150 micron diameter nylon fiber and detected by a laser Doppler vibrometer, versus pump-probe distance. The probe laser spot (diameter 150micron) position was kept fixed on a part of the sample that was covered by a small piece of retroreflective tape. The pump laser spot (diameter 150 micron) was scanned along the wire axis by means of a Newport translation stage that moved the mirror that guided the pump laser beam towards the wire.

We have performed a two dimensional Fourier transform on the measured dynamic displacement field signal $S(x, t)$, thus obtaining the signal amplitude $S(k, f)$ versus wavenumber $k$ and frequency $f$. It appears that the piezo-needle excitation was able to excite A0 Lamb waves only (see Figure 8 , bottom left) due to the fact that this type of excitation gives a very limited force acting as compression or tension through-the-thickness which was insufficient to excite S-modes at measurable levels. The maximum in the dispersion plot (Figure 8 , bottom left) at $2.5 \mathrm{MHz}$ in a wide range of wave numbers can be 
associated with a local resonance in the excitation spot. The guided wave velocity at $f_{\text {res }}=2.5 \mathrm{MHz}$ is about $\mathrm{k}_{\text {res }}=22 \mathrm{~km}^{-1}$, corresponding to a phase velocity of $0.7 \mathrm{~km} \cdot \mathrm{s}^{-1}$ and a wavelength $\lambda_{\text {res }}=2 \pi / \mathrm{k}_{\text {res }}=0.3 \mathrm{~mm}$ or about 2 times the diameter of the wire.

Figure 8 (bottom right) also shows a fit of the experimental data with an analytical infinite plate model for a plate of $150 \mu \mathrm{m}$ thickness. The fitted plate value was $Y_{\text {equivalent plate }}=(2.54 \pm 0.07) \mathrm{GPa}$. Using the geometrical shape adaptation factor of Table 1 (circle to plate correction of $15 \%$ ), we obtain a value $\mathrm{Y}_{\text {wire }}=(2.92 \pm 0.07) \mathrm{GPa}$.
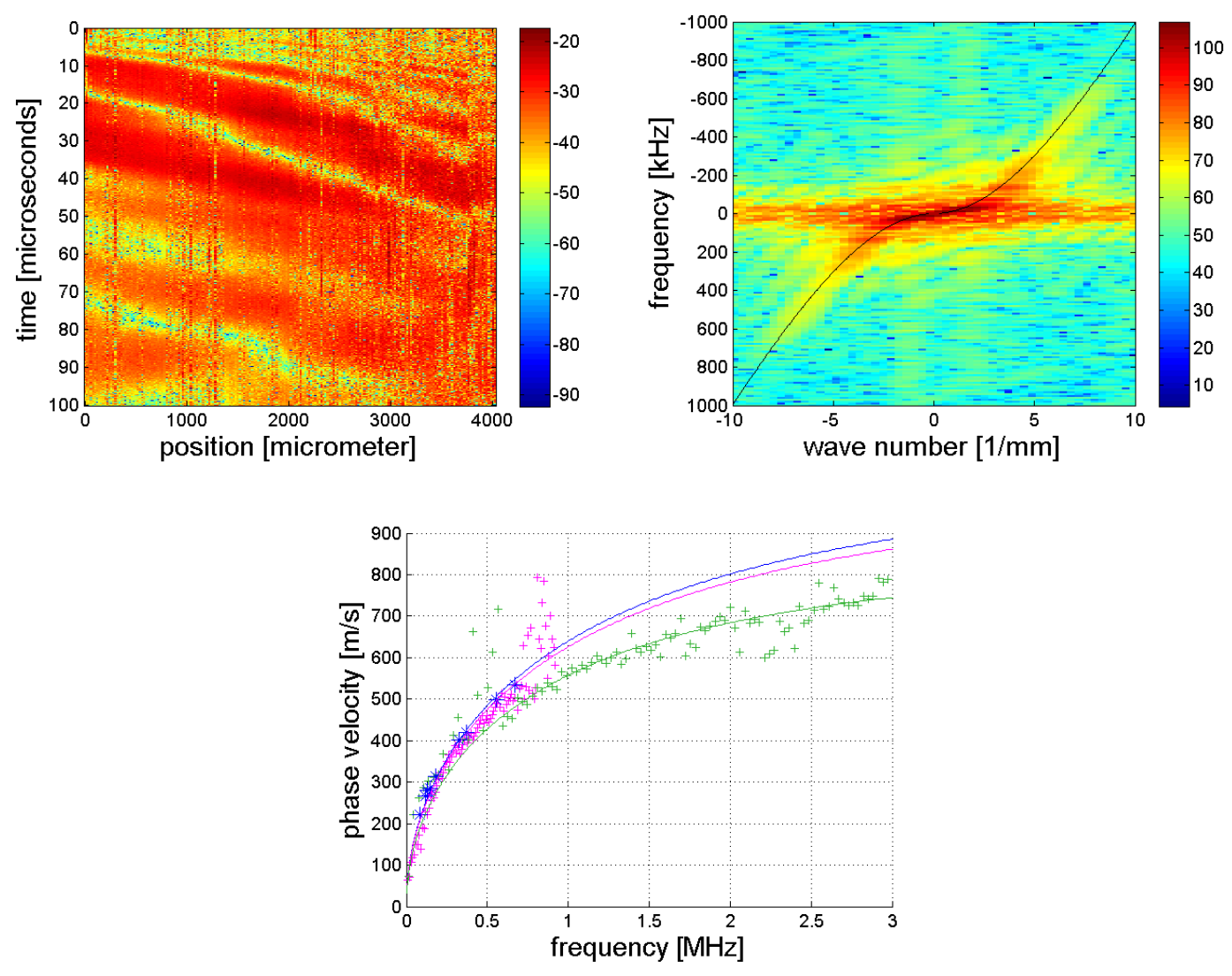

Figure 9: Top left: 2D color map (logarithmic (dB) of arbitrary units) representation of displacement signals, photoacoustically generated by focused laser pulses of 10 ns duration on a 150 micron diameter nylon fiber and detected by a laser Doppler vibrometer, versus pump-probe distance. Top right: dispersion spectrum of the displacement in $\mathrm{k}, \mathrm{f}$ domain. Bottom: corresponding phase velocity dispersion curve (cross symbols), fit with analytical infinite plate model (full line) of $150 \mu \mathrm{m}$ thickness and $Y_{\text {equivalent plate }}=(3.14 \pm 0.07) \mathrm{GPa}$, which after shape adaptation gives a value for the wire $Y_{\text {wire }}=(3.61 \pm 0.07) \mathrm{GPa}$. The solid lines show the fitted curves obtained in the piezo-needle experiment (blue), the nanosecond laser experiment (magenta) and the picosecond laser experiment (green).

We have followed the same approach using ns laser pulses (Figure 9). The probe laser spot (diameter 150micron) position was kept fixed on a part of the sample that was covered by a small piece of retroreflective tape. The pump laser spot (diameter 150 micron) was scanned along the wire axis by means of an APT Thorlabs translation stage that moved the mirror that guided the pump laser beam towards the wire. Fitting with an analytical infinite plate model (full line) of $150 \mu \mathrm{m}$ thickness yielded $Y_{\text {equivalent plate }}=(3.14 \pm 0.07) \mathrm{GPa}$, which after shape adaptation (15\% circle to plate) gives a value for the wire $Y_{\text {wire }}=(3.61 \pm 0.07) \mathrm{GPa}$. 
In summary, the piezo-needle experiment, the nanosecond laser scanning experiment and the picosecond laser scanning experiment yield the following respective values for Young's modulus of the wire: $Y_{\text {wire, piezo-needle }}=(4.5 \pm 0.1) \mathrm{GPa}$. $Y_{\text {wire,ps }}=(2.92 \pm 0.07) \mathrm{GPa}$. $\mathrm{Y}_{\text {wire, ns }}=(3.61 \pm 0.07) \mathrm{GPa}$. The respective fits are shown together in Figure 9 (bottom). On the basis of the small statistical errors on the values, the substantial differences between the obtained values infer that the measurements or analysis has been subject to configuration dependent systematic errors. One source of error can lie in the not perpendicular projection of the scanning direction of the translation stage guiding the pump or probe laser beam on the sample surface, introducing an error in the scanning step. Also the poor reflection of the light of the probing laser Doppler vibrometer causes a measurement uncertainty. Modification of the material properties due to laser heating in the ps and ns experiment is possible, but is not expected to affect the dispersion results in the case of probe scanning (out of the pump laser spot area). As shown above, in the accessed frequency range, possible effects of different wire tension can be neglected. Effects of using an ad hoc value for Poisson's ratio (0.4) in the fits are only expected for the high frequency part of the data (nature of propagation tending to the one of Rayleigh surface waves).

As mentioned above, extrapolated DMTA data predict values for Young's modulus of nylon between $5.7 \mathrm{GPa}$ and $6.4 \mathrm{GPa}$ in the $5 \mathrm{kHz}-1 \mathrm{MHz}$ range accessed in our experiments. The fitted values are systematically lower than this prediction, showing the limitations of the DMTA extrapolation. On one hand, neglecting dispersion in the analysis of the LDV experimental data can introduce an error in those. On the other hand, also the use of time-temperature superposition extrapolation of the DMTA data (sub-kHz range to $100 \mathrm{kHz}$ range) is subject to substantial uncertainties.

\subsection{Polyamide wire grid sample}

Although with the presented techniques local excitation and detection of guided elastic waves along fibers is possible, in complex structures such as in porous materials, the wave patterns along fibers and extracted dispersion curves are strongly affected by the finite length of those fibers.

When the pump-probe scanning path is kept within one strut of the frame of a porous material, and therefore shorter than the strut length, then the spatial Fourier transform performed to obtain the $k, \omega$ dispersion plot is affected by the finiteness of the window due to convolution of the k-spectra with the spectrum of the windowing function (e.g. sinc function in case of rectangular windowing). The change of geometry at the end of fibers, via a change of acoustic impedance, results also in the generation of reflected waves. In principle reflected waves are benign in terms of data analysis, as they add new branches in the dispersion plots that can be easily distinguished from the ones of the original wave (they are located in different quadrants). However, when damping is small and multiple reflections are strong, then standing waves occur, along with strong peaks at particular wavenumbers, making it sometimes difficult to extract the dispersion spectrum.

As an intermediate step between the simplicity of a long fiber and the complexity of a 3D porous network, we have performed scanning experiments on a 3D printed PA polymer grid with characteristic dimensions in the $\mathrm{mm}$-range (Figure 10). This has allowed us to perform and analyze scans along a 
single fiber within the large network, and to follow more complicated paths, along different fibers and across fiber-cross-connections.

Compared to real life porous networks, the investigated macroscopic PA grid offers the advantage of (i) having the same and therefore well-known elastic properties as bulk PA, (ii) having a substantial fiber diameter allowing for easy position scanning of the probe beam spot along the network, (iii) having fibers that are thick enough to withstand repeated impulsive laser excitation, (iv) having a periodical structure, which simplifies numerical calculations, and adds interesting phononic crystal features.

The Young's modulus of the polyamide was estimated by the supplier to be between 1 and 2GPa, with Poisson's ratio of about 0.4 and density $1200 \mathrm{~kg} \cdot \mathrm{m}^{-3}$.

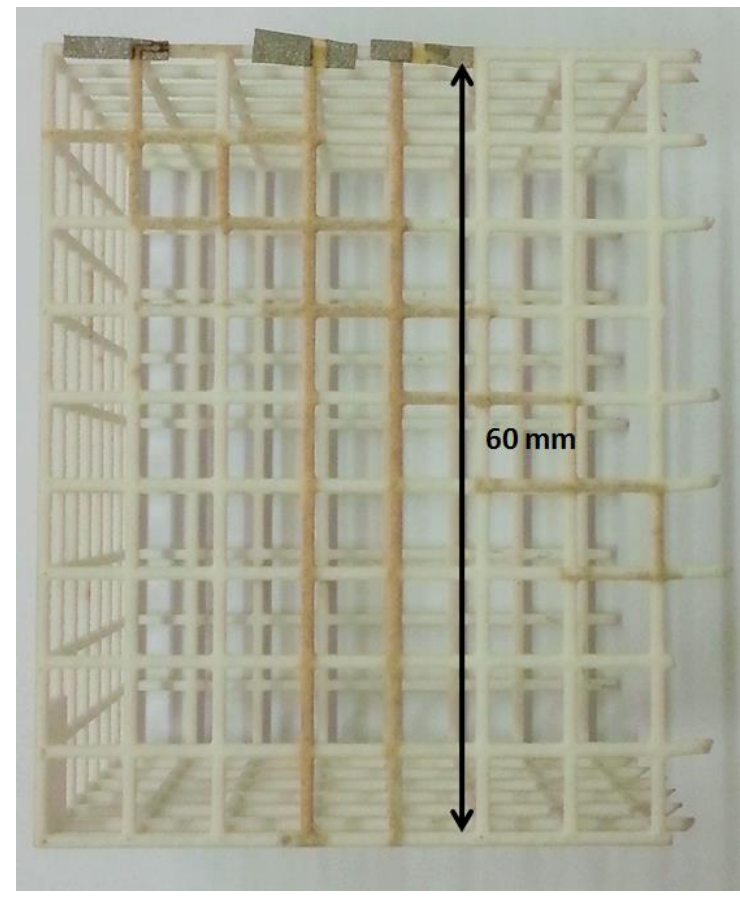

Figure 10: Polyamide fiber cubic grid sample, with average grid strut thickness $1.1 \mathrm{~mm}$ and distance between adjacent strut connections $7 \mathrm{~mm}$. Scanning measurement were performed in the whole length of $60 \mathrm{~mm}$ (from top to bottom) in scanning steps of $0.1 \mathrm{~mm}$.
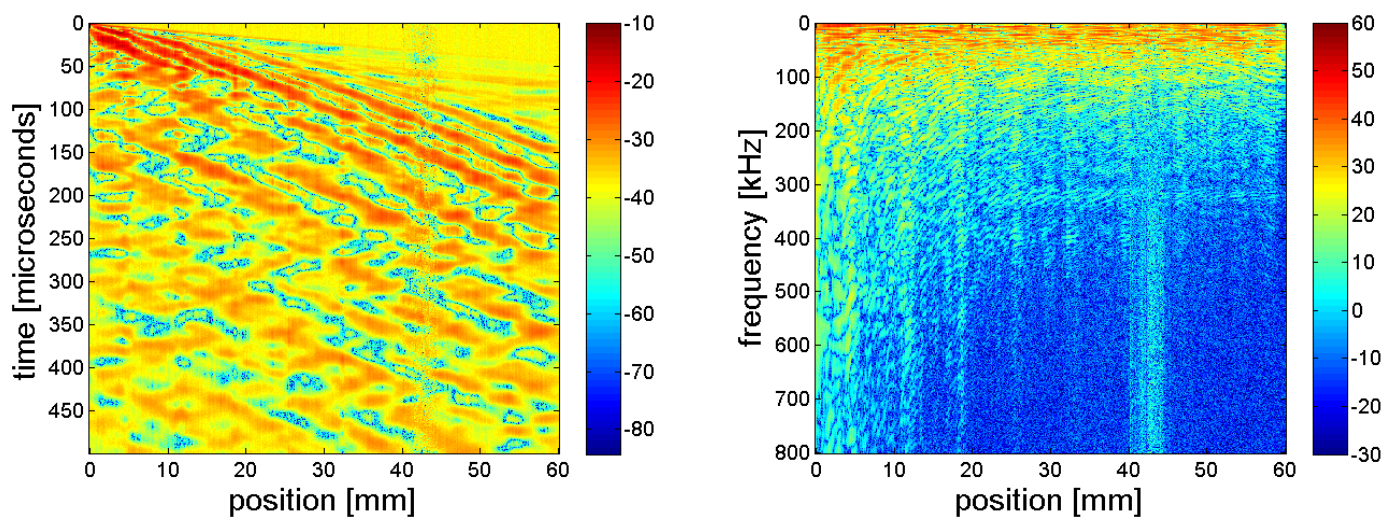

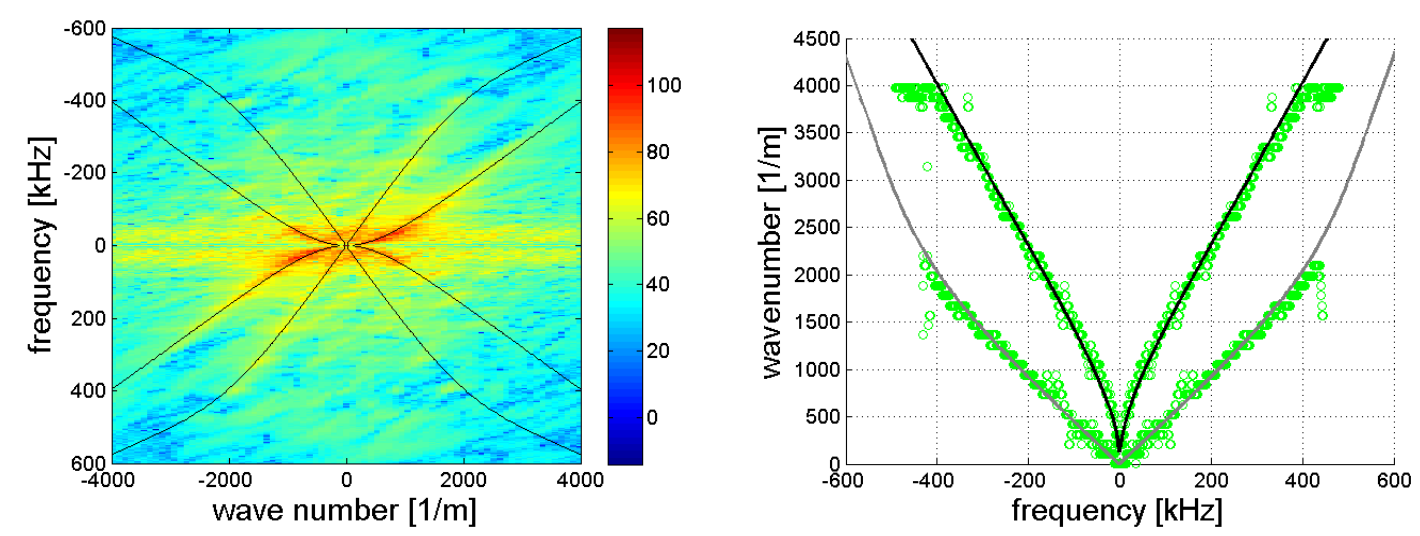

Figure 11: Top left: logarithmic amplitude of the magnitude of LDV detected time signals on the PA grid shown in Fig.10 for pump-probe distance between 0 and $60 \mathrm{~mm}$. Top right: signal amplitude spectra versus position. Bottom left: spectral amplitude versus frequency and wavenumber, obtained as the 2D Fourier transform of the dynamic displacement field $S(x, t)$. Bottom right: extracted dispersion curves for the right and left going $\mathrm{AO}$ and $\mathrm{SO}$ Lamb modes that travel along the strut.

Figure 11 shows the result of an LDV position scan along one of the struts in the grid of photoacoustically excited waves. The full lines represent the best fits of the dispersion curves, fitting Young's modulus while keeping a Poisson's ratio of 0.4 and a density of $1200 \mathrm{~kg} \cdot \mathrm{m}^{-3}$, fit with analytical infinite plate model (full line) of $1.1 \mathrm{~mm}$ thickness and $Y_{\text {equivalent plate }}=(1.83 \pm 0.01) \mathrm{GPa}$, which after shape adaptation $(+5 \%)$ gives a value for the wire $Y_{\text {wire }}=(1.92 \pm 0.01) \mathrm{GPa}$ for $A 0$ mode and and $Y_{\text {equivalent plate }}=(2.03 \pm 0.05) \mathrm{GPa}$, which after shape adaptation $(+5 \%)$ gives a value for the wire $\mathrm{Y}_{\text {wire }}=(2.13 \pm 0.05) \mathrm{GPa}$ for $\mathrm{SO}$ mode. The two distinguished first arriving wave fronts in the color map of the signal magnitude reveal the presence of two guided wave modes, which is confirmed by the AO- and SO-like Lamb wave dispersion branches in the phase velocity dispersion plots. Whilst the piezo-needle excitation was able to excite AO Lamb waves only, the photoacoustic excitation could excite both Lamb waves as a result. Fitting the Young's modulus while keeping a Poisson's ratio of 0.4 and a density of $1200 \mathrm{~kg} . \mathrm{m}-3$ led to a value $Y=(1.83 \pm 0.01) \mathrm{GPa}$, which after shape adaptation $(+5 \%)$ gives a value for the wire $Y_{\text {wire }}=(1.92 \pm 0.01) \mathrm{GPa}$ for the $\mathrm{A} 0$ mode and $\mathrm{Y}=(2.03$ $\pm 0.05) \mathrm{GPa}$, which after shape adaptation $(+5 \%)$ gives a value for the wire $Y_{\text {wire }}=(2.13 \pm 0.05) \mathrm{GPa}$ for the S0 mode, which is in the expected range.

Interestingly, the amplitude of the displacement is found to be enhanced, in particular for high frequencies, where the scanning position is trespassing a strut cross connection (every $7 \mathrm{~mm}$ ). This effect, which was not present in numerical simulations, can possibly be attributed to an increased excitation efficiency due to improved local wave recoil at the strut cross connections.

The position dependence of the amplitude spectra of the displacements, and the extracted 1/e damping distance (Figure 12a), reveal that the attenuation of the waves is increasing with frequency in the $0-500 \mathrm{kHz}$ range. Figure $12 \mathrm{~b}$ shows the frequency dependence of the loss angle $\psi$, extracted from the real value and from the damping distance by assuming an $\mathrm{AO}$ Lamb wave displacement evolution $S(\omega, t)=S_{0} \exp \left(i \omega t-i k_{R} x-k_{1} x\right)$, with $\omega=2 \pi f$ the angular frequency, $x$ the pump-probe distance, $S_{0}$ the wave displacement amplitude at $\mathrm{x}=0, \mathrm{k}_{\mathrm{R}}$ and $\mathrm{k}_{\mathrm{l}}$ the real and imaginary part of the wavenumber respectively, and $\operatorname{tg} \psi=\mathrm{k}_{1} / \mathrm{k}_{\mathrm{R}}$.

Intrinsically, the followed approach of mapping the spatiotemporal behavior of guided waves allows to unambiguously extract information on the imaginary part of the Young's modulus from the 
(frequency dependent) degree of damping of the waves with distance. However, in the case of nylon, DMTA analysis showed that the damping in the accessible frequency range was only of the order of $2 \%$. The uncertainty on the determined dispersion data was too high to resolve this. Material damping is expected to be similarly low in the polyamide. Therefore, the experimentally observed substantial attenuation of the guided waves in the PA grid should not be ascribed to material damping, but it is a result of the multiple reflections that the guided waves undergo at every strut connection, leading to weakly diffusive type of wave propagation. Though not representative for the material properties, this effective damping is very interesting for models that estimate macroscopic behavior from this kind of microscopic measurements.
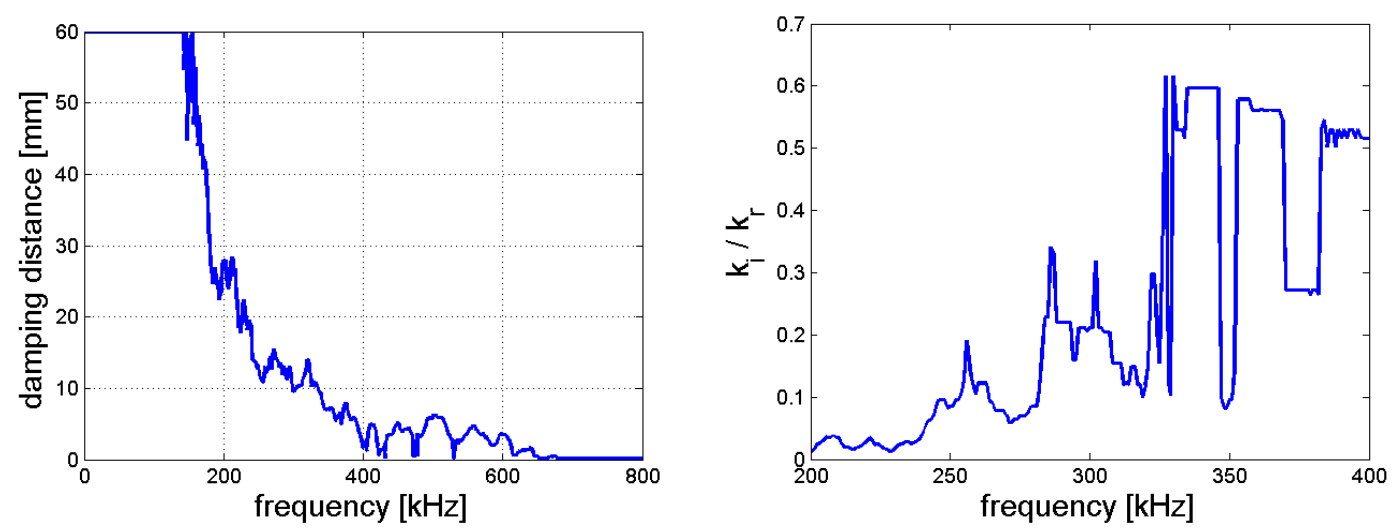

Figure 12: Left: Frequency dependence of the 1/e damping distance for the PA grid. Right: Frequency dependence of the loss factor $\operatorname{tg}(\psi)=k_{1} / k_{R}$ for the PA grid
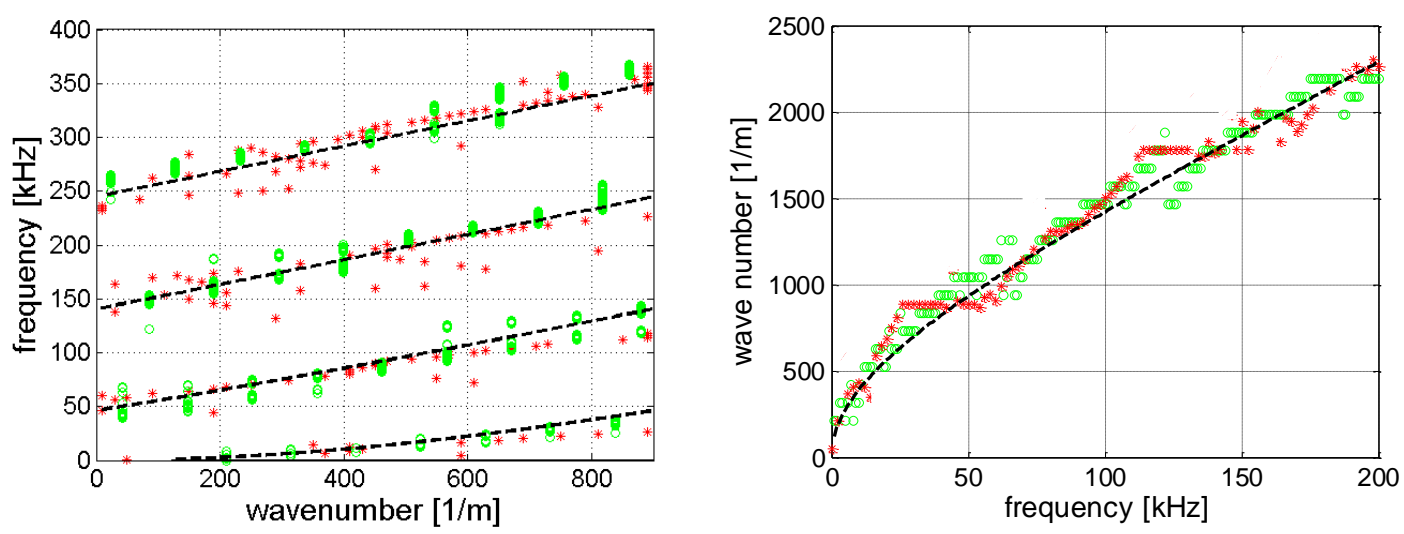

Figure 13: Left: dispersion curves (AO mode) in $k$, $f$ domain in the Brillouin zone of the PA grid phononic crystal for the experimental data (green circles), numerical model (red asterisks) and analytical model of an infinite plate with a Young's modulus of 1.8GPa. Right: non-shifted wavenumber as function of frequency.

Figure 13 (left) shows the evolution of wavenumber on horizontal axis as function of frequency on vertical axis for the experimental measurement on PA grid, also shown in Figure 11 (bottom right), for an analytical infinite plate model with a Young's modulus 1.8 GPa (black dashed line), Poisson's ratio of 0.4 and density of $1200 \mathrm{~kg} / \mathrm{m}^{3}$ and for a numerical finite element model of the PA grid representing only two struts connected in the middle point, forming a simple cross of $L=7 \mathrm{~mm}$ length for both branches and $\mathrm{h}=1.1 \mathrm{~mm}$ thickness. Figure 13a shows the results for wavenumber values between 0 
and $k_{\max }=2 \pi / L$ with all higher $k$-values properly shifted by $2^{*} \pi^{*} n$. Figure $13 \mathrm{~b}$ shows the same results, without shifting $k$. Both figures (but more easily visible in Figure 13b) show typical S-shape curves corresponding to wavenumber band gaps at values around the edges of the Brillouin zone, i.e. around $k_{\max }=2 \pi / L=897 \mathrm{~m}^{-1}$ and multiples of this value.

A simple point excitation in the out of plane direction was used at one strut end of the numerical finite element model and Bloch-Floquet [12][13] boundary conditions were applied at each termination. A parametric study was performed scanning frequency and wavenumbers values. From this computation, the average absolute value of the out of plane displacement, as a function of wavenumber and frequency, of one face was extracted, and a search for one peak wavenumber per frequency was performed.

The calculations considered a Young's modulus of 1.8GPa, a value in the range of what was expected by supplier (1-2 GPa), a Poisson's ratio of 0.4 and a density of $1200 \mathrm{~kg} / \mathrm{m}^{3}$. The dispersion curve obtained using a model with Bloch-Floquet boundary conditions (red stars) turns of to be corresponding well with the one obtained by an analytical infinite plate model (black dashed line). Both models correspond well with the experimental data of the PA grid (green circles).

Effects of phononic band gaps at the edge of the Brillouin zone are better visible in the numerical calculations than in the experimental data, probably due to the finiteness of the experimentally scanned spatial window.

\section{Conclusion}

Detection by laser Doppler vibrometry of guided elastic waves excited by a piezo-needle actuator (resonant excitation AO Lamb waves) and by the photoacoustic excitation(wide band excitation of $\mathrm{AO}$ and SO Lamb wave) allow to determine the wave dispersion and damping in the $5 \mathrm{kHz}-1 \mathrm{MHz}$ range. The approach was demonstrated for a 150micron diameter nylon wire and for a cubic wire grid consisting of interconnected polyamide struts of $1.1 \mathrm{~mm}$ diameter and $7 \mathrm{~mm}$ length. For the photoacoustic measurements, both a pump and probe laser spot scanning scheme were implemented. Wave reflections due to strut interconnections in the polyamide wire grid turn out not to substantially disturb the spatiotemporal dependence of the $\mathrm{A} 0$ and $\mathrm{SO}$ Lamb wave displacement pattern along a grid wire crossing 8 interconnections along a scanning path of $60 \mathrm{~mm}$.

DMTA measurements on the nylon wire predict a weak dispersion of Young's modulus in the frequency range covered by the LDV experiments. Therefore, although the approach allows to extract a frequency dependent Young's modulus, for the sake of robustness of the fit, we have assumed a frequency independent value for both the nylon wire and the PA cubic wire grid sample. Fitting the velocity dispersion data with a semi-analytical plate model and applying a geometrical adaptation factor to account for the circular wire shape resulted in an average Young's modulus value of $Y_{\text {nylon }}$ wire $=(3.7 \pm 0.8) \mathrm{GPa}$. This value is lower than values around 6GPa predicted by DMTA, probably as a result of uncertainties in the used time-temperature extrapolation. 
A Bloch-Floquet model successfully accounted for effects on the numerical predictions for the spatiotemporal dependence of the flexural wave displacement pattern caused by the strut interconnections in the polyamide wire grid.

The results infer that the two LDV-approaches should also be feasible to characterize the elastic properties of polymer struts in complex foam networks with strut lengths in the sub-cm and sub-mm range. The demonstrated feasibility of extracting a good quality dispersion curve (and fitted Young's modulus) from a pump-probe scan across several strut-interconnections gives a positive perspective for scanning across struts in a complex porous material frame. Additional work is needed to give better consistency between results obtained piezo-needle, nanosecond laser and picosecond laser excitation.

The implementation of the approach for determining the temperature and dependence of the real and imaginary part elastic moduli is ongoing. 


\section{References}

[1] "A method for characterisation of the static elastic properties of the porous frame of orthotropic open-cell foams", C. Van der Kelen, J. Cuenca, P. Goransson, International Journal of Engineering Science 86 pp. 44-59 (2015)

[2] "On the influence of frequency-dependent elastic properties in vibro-acoustic modelling of porous materials under structural excitation", C. Van der Kelen, P. Goransson, B. Pluymers, W. Desmet, Journal of Sound and Vibration, vol. 333, Issue 24, pp. 6560-6571, (2014)

[3] "Elastic and damping characterization methods of acoustic porous materials: Available experimental methods and application to melamine foams (review article)," L. Jaouen, A. Renault, and M. Deverge, Appl. Acoust. 69, 1129-1140 (2008).

[4] "Characterisation of elastic parameters of acoustical porous materials from beam bending vibrations," A. Renault, L. Jaouen, and F. Sgard, J. Sound Vib. 330, 1950-1963 (2011)

[5] "Time-temperature superposition in viscous liquids", N. B. Olsen, T. Christensen and J. C. Dyre, Physical Review Letters 86 (7), 1271-1274 (2001).

[6] "Long-term stress relaxation prediction for elastomers using the time-temperature superposition method", S. Ronan, T. Alshuth, S. Jerrams, N. Murphy ; Materials and Design 28 (2007) 1513-1523

[7] "Temperature and frequency dependence of the visco-elasticity of a poro-elastic layer", C. Glorieux, J. Descheemaeker, J. Vandenbroeck, J. P. Groby, L. Boeckx, P. Khurana and N. B. Roozen, Applied Acoustics 83, 123-126 (2014).

[8] "Generalization of the Williams-Landel-Ferry equation", L. Dagdug, L.S. Garcia-Colin,Physica A, 250, 133( 1998).

[9] "Vibration in continuous media”, Jean Louis Guyader, ISTE LtD, London, UK, ISBN 978-1-905209-279 (2006)

[10] "Dispersion relations for elastic waves in plates and rods", A.F.F. Amirkulova, PhD thesis, Rutgers, The State University of New Jersey (2011)

[11] "Acoustique des instruments de musique", A. Chaigne, J. Kergomard, Ed. Belin, 2008.

[12] "Floquet waves and classical plane waves in an anisotropic periodically multilayered medium: application to the validity domain of homogenization", C. Potel, J.-F. de Belleval, and Y. Gargouri, The Journal of the Acoustical Society of America, vol. 97, no. 5, p. 2815 
[13] "Analysis of the propagation of plane acoustic waves in passive periodic materials using the finite element method", P. Langlet, A.-C. Hladky-Hennion, and J.-N. Decarpigny, J. Acoust. Soc. Amer., vol. 98, no. 5, pp. 2792-2800, 1995

\section{Acknowledgements}

The authors acknowledge the R\&D team at Huntsman, Everberg, Belgium for very fruitful scientific discussions and for providing the polyamide grid sample.

This article profits from fruitful interactions with partners in COST Action DENORMS (CA15125), supported by COST (EuropeanCooperation in Science and Technology)". 
REVIEW:

The authors appreciate very much the thorough reading by the reviewers, and their relevant and constructive comments and suggestions, which we have now incorporated in the revised version to a maximum extent. Below we respond to all reviewers' comments and specify how we have taken those into account in the revision.

Reviewers' comments:

Reviewer \#1: This paper is related to characterization of polymer fiber grid by laser Doppler vibrometry LDV. The topic investigated by authors and connected with characterization of elastic properties of porous materials is very important. Authors have made lot of good experimental as well as numerical investigations. However, in my opinion this paper was not prepared conscientiously and cannot be published in the present form. This paper looks rather like report from some project related research. Please find my critical comments bellow.

1. Authors have prepared section with highlights as it is requested by Journal Optics and Lasers in Engineering but for me its not clear what is really new. There is only introduction in section 1 without state-of-the-art with clear underlined what was done in this field and what is still not solved. Authors have not clearly underlined what is new related to topic literature.

\section{Response:}

To make clear what is new in this article, we have added a paragraph at the end of section 1:

"This paper presents new experimentally obtained dispersion relationships of fiber wires and fiber grids, which are compared with numerically obtained results. These results are relevant in the context of the overall objective of this work to make a step towards microscale characterization of foam structures. "

2. Authors have cited only nine literature positions and they are relatively old. Authors need to prepare improved state of the art with emphasis what new was done.

Response:

New literature references have been added on the state of the art of research in this field.

3. Something is wrong with figures numeration. Figure 2 appears in Highlights section as well as in section 2 with experimental details.

Response:

Figure numeration has been checked and a distinct numbering is used for Highlights and Article.

4. For me the intention of authors is not clear. Figure 1 is located in Highlights but reference to 
this figure is located in section 2. In my opinion all figures should be place directly in the paper unless Highlights will be a part of the paper.

Response:Figure 1 in the highlights (now renamed to Fig H1) is also contained in the manuscript. To be more precise in Figure 1 and Figure 10 of this revision. Figure 1 of this revision shows the test setup of the nylon wire, Figure 10 of this revision shows the polyurethane fiber cubic grid sample. The intention of this paper is to experimentally characterize the elastic properties of polymer fibers by means of laser Doppler vibrometry.

To strengthen the paper to this respect, we modified the second last paragraph of section 1 into

"The intention of this paper is to characterize polymer fibers by means of laser Doppler vibrometry. The approach presented in this paper is applied to a nylon fiber with a diameter of approximately $100 \mu \mathrm{m}$ and a rectangular polyurethane fiber grid with dimensions in the $0.1 \mathrm{~mm}-100 \mathrm{~mm}$ range. These samples were chosen on the basis of the similarity of their geometry with the one of struts and networks in foams, while having dimensions that are larger, making the experimental proof of concept of the proposed approach more simple. "

In addition, we have modified the title of the paper into

Elastic characterization of polymer fibers by laser Doppler vibrometry

5. Descriptions of figures are too long and need to be shortened (for example fig.9, fig.10).

Response:

Where possible, the captions of the figures have been shortened.

6. Figure 3 displacement achieves the amplitude above $1000 \mathrm{~mm}(1 \mathrm{~m})$ is it correct? I think that this is simply mistake and should be $10^{\wedge}(-2)$ not $10^{\wedge} 2$

Response:

Scale of current figure number 2 (old fig number 3) has been corrected to $1000 \mathrm{~nm}$.

7. Page $3-\mathrm{kHz}-100 \mathrm{kHz}$ should it be $1 \mathrm{kHz}-100 \mathrm{kHz}$ ?

Response:

Correction has been made to $1 \mathrm{kHz}-100 \mathrm{kHz}$

8. Figure $4 \mathrm{x}$-axis frequency should it be from audio frequency (single $\mathrm{Hz}$ or $5 \mathrm{kHz}$ according information given in page 7) till $\mathrm{MHz}(1 \mathrm{MHz}$ see page 7$)$ ? There is for example $10^{\wedge}(-20) \mathrm{Hz}$ on $\mathrm{x}$ axis. Bottom of page 5 is also information few $\mathrm{kHz}-\mathrm{MHz}$ but in the range indicated by red lines.

Response:

Figure 4 shows the DMTA measurement results. The DMTA results are now presented in two subfigures. Fig 4a shows the dependency of the Young's modulus as function of temperature (with fixed excitation frequency). Fig $4 b$ shows the results converted into frequency-dependency (with fixed temperature), in which we now only show the useable part of the frequency range. We believe that this way of presenting the DMTA-data is more appropriate. 
9. Page 8 - table1 is it description just above the table? It look like normal text. However, if it is, it should be shortened.

Response:

We have shortened the legend of Table T1. The body text contains all necessary information.

10. Page 15 in the description of fig 12, there is Fig.X11. Should it be Fig.11?

Response:

Indeed, correction has been made to Fig.11

11. Page 16 Figure $\mathrm{X} 12$ should it be Figure 12

Response:

Indeed, correction has been made to Fig.12

12. Page 8 reference to eq. 1 in the text. There are eq. $1 \mathrm{a}$ and eq. $1 \mathrm{~b}$ it need to be clarify which one is referenced to.

Response:

Correction has been made to eq. $1 a$

13. Authors have investigated different frequency range for piezo-based excitation (up to $\sim 1.1 \mathrm{MHz}$ ) and laser-based excitation (up to $3 \mathrm{MHz}$ ). Have authors investigated higher frequencies for piezoelectric transducer? There should be other resonances even around $4.5 \mathrm{MHz}$ (thickness mode) depending on piezoelectric material properties. Authors do not need to extend this research for higher frequencies but some explanations why different frequency range were utilized should be given in the paper.

Response:

Thank you for this comment. The following sentence (page 3) has been extended (new text underlined)

By applying a sinusoidal voltage with 50Volt amplitude, generated by a computer controlled homemade sine wave generator and amplified by a power generator ENI AP4OOB, bending modes of the bilayer membrane could be efficiently excited, with maximum displacement amplitudes at resonances in the $50 \mathrm{kHz}-700 \mathrm{kHz}$ range ranging between $60 \mathrm{~nm}$ and $1600 \mathrm{~nm}$. The reason to limit the frequency range to the range mentioned is that the structural resonances of the piezo-element and its membrane lie in this range.

14. Authors wrote that piezo based excitation is able to excite only A0 mode. I suppose this is due to the fact bonding mode vibration of membrane. What is about with amplitude of S0 mode, this mode should still exists, but its amplitude is very low in comparison to A0. It need to be commented. 
Response:

On page 12 we have added the following text to comment on this issue:

"It appears that the piezo-needle excitation was able to excite AO Lamb waves only (see Figure 8, bottom left) due to the fact that this type of excitation gives a very limited force acting as compression or tension through-the-thickness which was insufficient to excite S-modes at measurable levels"

On page 17 we have added the text

"Whilst the piezo-needle excitation was able to excite AO Lamb waves only, the photoacoustic excitation could excite both Lamb waves as a result."

Before publication of this paper it need to be clearly underlined what new was done in this research.

Response:

The novelty of the presented research is implicitly summarized in the conclusion of the article.

To the best of the authors' knowledge, no previous comparisons have been made between DMTA, piezo ultrasonics and laser ultrasonics results on polymer fibers. With this article, we present a first cross-validation of the 3 techniques, and show results that give a promising perspective for their use for wide bandwidth characterization of the frequency dependent elastic moduli.

The concise study on the influence of the fiber shape on the dispersion gives a useful guideline to the $R \& D$ community that is involved in fiber characterization, without having access to complicated analytical calculations or heavy numerical toolboxes.

Moreover paper need to be substantially improved according the comments.

Reviewer \#2: The paper presents interesting experimental study on Young's modulus estimation (based on fitting dispersion curve) for polymer fibers using laser Doppler vibrometry.

In my opinion paper should be interesting for OLE readers, however it needs some corrections especially on editorial part.

- The figures and table captions are much too long. The detailed description should be move into the text part.

Response:

Where possible, the captions of the figures have been shortened.

- There is no Figure 1 (as I understand authors refers to the Figure 1 in Highlights)

Response:

Figure numeration has been corrected. 
- Authors refer to e.g. Figure 2a. There is only Figure 2. In other place author refer to Figure X(left). All the Figures should be divided into a), b) ... and all reference in the text should be unify correspondingly.

Response:

Figure numeration has been corrected.

- Some Figures like 8, 10 are missing $Y$ axis labels.

Response:

Figures (current figure numbers 7 and 8) have been modified and explanations have been added to better understand the $Y$-axis on those figures.

- "wave number" should be replace with "wavenumber" (e.g. Figure 12).

Response:

Wavenumber has been corrected accordingly.

- negative frequency part could be removed from figure

Response:

For now, we have not followed this advice, for the reason that the positive/negative frequencies actually correspond to right/left going waves. There is no redundant information. The fact that they give very similar results supports their validity.

- Unify the all the units (In Figure 12 there in both [millimeter] and [mm]

Response:

Figure 12 has been modified accordingly : [mm]

- Standardize the notation of values in text. One may found "10mm" and "10 mm".

Response:

Notations of numbers with units have been checked and put in the same notation like "10mm".

Please check the paper carefully for other mistakes as well because it was obviously not proofread enough.

Reviewer \#3: Though the article itself is well structured and written in a clear manner and the topic of 
is of great demand, there are several remarks and questions which should be clarified, which are the following

1) Figure titles are too long (Fig. 2, 4, 5, 6, etc). The information provided there should be given in the manuscript text.

Response:

Where possible, the captions of the figures have been shortened.

2) Page No. 4, last paragraph: the authors mention that "The phase velocity dispersion curve, ..., was extracted from the axial position dependence of the displacement signals". However, no details are provided about the approach which was utilized for this extraction. Though further in the text (Page 11 , second paragraph) it is mentioned that 2D Fourier transform is applied, it might be more convenient to give the appropriate references and description here, on page 4.

Response:

On page 4 the procedure is outlined better, by adding the following sentence:

"A Fourier transform was used to compute the response of the wire in the frequency domain."

3) Page 6, last paragraph. What does "imaginary part of wave induced displacement pattern" mean? How the authors managed to obtain this data from the experimental LDV measurements?

Response:

The results shown in Fig 4 (page 6) are frequency domain results, showing the frequency of excitation only. This fact is now explicitly mentioned in the text on page 6 (new text underlined):

Figure 4 shows the frequency domain results (real and imaginary parts) of the wave induced displacement pattern along the axial direction at different discrete, piezo-needle excitation frequencies.

On page 4 the procedure is outlined better, by adding the following sentence:

"A Fourier transform was used to compute the response of the wire in the frequency domain."

4) Page 7, first paragraph. The authors mention that along with the FEM simulations for a wire with an arbitrary cross-section they use an infinite plate model, explaining it by a model simplicity. However, the utilization of the latter approach (plate model) requires an introduction of a non-natural shape adaption factor, depending on the shape of an initial wire. Current advances in numerical methods for elastodynamic simulation of prolonged bodies with a finite cross section make the evaluation of their dispersion properties is rather straightforward and does not require heavy computations. Therefore, the necessity to utilize the plate model is a question for a discussion. Another question, arising here, is whether the discussion and results concerning this shape adaption factor fit the journal topics. 
Response:

Although indeed numerical methods have become very adequate and powerful, the availability of a simplified analytical model, in combination with a reliable shape adaptation factor does have the important advantage that, compared to an approach in which the direct calculation is performed numerically it can be very easily implemented (programmed) in a multiparameter fitting problem, with fast calculations. In addition, we (and hopefully also some readers) found (will find) it interesting to find out in which way the shape and cross-section of different fiber shapes can be made to match.

We have added the following sentence to page 7 of the manuscript:

"This has the important advantage in that, compared to an approach in which the direct calculation is performed numerically, a semi-analytical model for a plate can be very easily implemented (programmed) in a multiparameter fitting problem, with fast calculations."

5) The provided results of the wire Young modulus reconstruction vary strongly depending on the experimental approach used by the authors and are sufficiently lower than the DMTA obtained data. Unfortunately, no reliable explanation of such a behavior is given. Therefore, it might be useful to verify at first the developed experimental approaches over plate-like metallic (e.g., aluminium) samples, where the dispersion data and elastic moduli are well-known.

\section{Response:}

We agree that the difference in resulting Young's modulus of the nylon wire (Ywire, piezo-needle $=(4.5$ $\pm 0.1) \mathrm{GPa}$. Ywire, $p s=(2.92 \pm 0.07) \mathrm{GPa}$. Ywire, $n s=(3.61 \pm 0.07) \mathrm{GPa}$. ) between the different approaches impedes full satisfaction over the results. However, this is to a great extent due to the difficult nature of the sample, having quite strong damping and giving poor reflection of the light of the probing laser Doppler vibrometer. Nevertheless, we believe that the results are still very valuable, showing that:

- Young's modulus characterization up to $2.5 \mathrm{MHz}$ is feasible, albeit with a substantial uncertainty (Y-range (2.9-4.5)GPa)

- The Young's modulus at high frequencies is substantially smaller than the Young's modulus extrapolated from low frequency measurements. In our opinion this indicates the limitations of the extrapolation, which is an important message to material scientists using DTMA.

We have discussed in detail possible causes of this discrepancy on page 14 of the manuscript.

For completeness, we mention that photoacoustic excitation of waves in combination with scanning the displacements by interferometric techniques has been proven to be very adequate for elastic characterization of plates, with small error bars on the extracted elastic parameters. Some illustrative references from our research group are:

- "Investigation of elastic properties of CVD-diamond films using the lowest order flexural leaky lamb wave", K. Van de Rostyne, C. Glorieux, W. Gao, V. Gusev, M. Nesladek, W. Lauriks and J. Thoen, Physica Status Solidi (a) 172(1), 105-111(1999) 
- "Experimental investigation of leaky Lamb modes by an optically induced grating", K. Van de Rostyne, C.Glorieux, W.Gao, W.Lauriks and J.Thoen, IEEE Transactions on UFFC 49(9), 12451253 (2002)

- "Laser ultrasonic study of Lamb waves: determination of the thickness and velocities of a thin plate", W.Gao, C.Glorieux, J.Thoen, International Journal of Engineering Science 41, 219228(2003)

- "Two-dimensional Gabor analysis of space-time transient Lamb waves using laser ultrasonic investigation", L. Martinez, N. Van Riet, C. Glorieux, Journal of the Acoustical Society of America 115(5), 2613(2004)

- "Guided elastic waves in porous materials saturated by air under Lamb conditions", Laurens Boeckx, Philippe Leclaire, Poonam Khurana, Christ Glorieux, Walter Lauriks and Jean-Francois Allard, Journal of Applied Physics 97, 094911(1-9) (2005)

- "Effect of loading a plate with different liquids on the propagation of Lamb-like waves studied by laser ultrasonics", X. XU, J. Goossens, G. Shkerdin and C. Glorieux, IEEE Transactions on UFFC, 55 (3), 675-85 (2008))

- "Investigation of the phase velocities of guided acoustic waves in soft porous layers", Laurens Boeckx, Philippe Leclaire, Poonam Khurana, Christ Glorieux, Walter Lauriks and Jean François Allard, Journal of the Acoustical Society of America 117(2), 545-554 (2005)

6) Page 16, last paragraph. The figure 15 is mentioned, however, not provided.

Response:

Correction has been made to the appropriate numbering.

7) It is a question for a discussion, whether the second specimen (3D printed cubic grid sample) is a reliable and an adequate model for the foam-like materials mentioned in the introduction. I would suggest the authors to pay more attention on this question, providing, may be, some references to the research where this problem has been investigated.

Response:

We agree that the 3D printed scale model is a strong simplification of a daily life porous material. However, it does present some very similar issues that would be encountered when doing measurements on a porous sample, with the goal of determining the Young's modulus of a strut:

- In both cases the waves are dispersive, which on one hand makes the measured wave propagation characteristics more rich in information, but on the other hand, makes the analysis complex in case of a frequency dependent modulus (for the 3D printed sample this could be realized in a next step, by heating the sample above it's glass transition temperature).

- In both cases the analysis is complicated by the finite length of the interconnected struts, which hampers the extraction of information for wavelengths that are longer than the strut length.

In the manuscript, the motivation to use a nylon fiber with a diameter of approximately $100 \mu m$ and a rectangular polyamide fiber grid with dimensions in the $0.1 \mathrm{~mm}-100 \mathrm{~mm}$ range, is given on page 2 
(2nd and 3rd paragraph). An additional paragraph was written (page 2, end of the introduction) which reads as follows:

"These samples were chosen on the basis of the similarity of their geometry with the one of struts and networks in foams, while having dimensions that are larger, making the experimental proof of concept of the proposed approach more simple. The dispersion curves are analyzed with respect to the influence of the shape of the fiber cross section and of the complex structure in which the fiber of interest is embedded. Although much simpler, similar to foams, the cubic grid is characterized by cross-connections, which result in discontinuities along the scanning path when the path is chosen long enough to allow to analyse long wavelengths and low frequencies. The next step towards realistic foams, using a 3D printed polymer grid with randomly oriented and connected struts, will be the subject of future investigations."

8) As it is mentioned in the introduction the general goal of the proposed research is to obtain some global elastic properties of foam-like materials in a non-destructive way. Therefore, a question arises whether the Young's modulus of a single strut in a grid would allow the estimation of these global properties.

Response:

This question is actually one of the main motivations of our current research, as explained in the introduction of the manuscript. In summary we try to

- Determine the local Young's modulus and Poisson ratio within individual struts

- Extract the effective macroscopic elastic parameters from numerical models that include those values as well as the foam morphology and that simulate the propagation of waves with wavelengths that span a large number of porous cells.

- Compare those parameters with the effective macroscopic parameters found from experiments, as determined e.g. as in "Investigation of the phase velocities of guided acoustic waves in soft porous layers", Laurens Boeckx, Philippe Leclaire, Poonam Khurana, Christ Glorieux, Walter Lauriks en Jean François Allard, Journal of the Acoustical Society of America 117(2), 545-554 (2005)

The main challenges are:

- determining Young's modulus at frequencies for which the wavelength is longer than the strut length

- unraveling the frequency dependence of Young's modulus from the guided wave dispersion, that also contains strong dispersion due to the struts being thinner than the acoustic wavelength

This article presents the result of one step in this challenging but exciting journey.

We have elaborated this further on page 1 in the following added paragraph:

"This can be achieved by numerical (finite element) modeling, in which the found microscopic elastic properties are used for the microscopic elements of discretized morphological foam structures that are obtained by microCT. The vibrational response of the macroscopic structure is then obtained by 
mechanically exciting it at the subsonic and audio frequencies of interest, for which the wavelength is much larger than the pore size. From the dynamically changing macroscopic strain map, the compressibility of the effective medium, or the wavelength of the travelling waves can be determined. "I 